\title{
Optical resolution of light engine based on InGaN/GaN nanoLED arrays: toward a superresolved light source
}

\author{
K. Kluczyk-Korch ${ }^{\dagger, *}$, A. Di Carlo ${ }^{\dagger, \ddagger}$, M. Auf der Maur ${ }^{\dagger}$ \\ ${ }^{\dagger}$ Department of Electronic Engineering, University of Rome Tor Vergata, 00133, Rome, Italy \\ $\S$ ISM-CNR, Via del Fosso del Cavaliere 100, 00133 Rome, Italy \\ *Email: katarzyna.kluczyk@uniroma2.it
}

\begin{abstract}
We present the optical simulations of a novel illumination source based on nanoLED arrays for the possible application in superresolved microscopy. We are simulating the images of various gold nanoparticle arrangements collected by the proposed nanoillumination microscope. The images are then analysed in order to understand the capabilities and limitations of the microscope.
\end{abstract}

Index Terms-nanoLED arrays, superresolution, optical microscopy

\section{INTRODUCTION}

There exist a number of different approaches to overcome the diffraction limit imposed by Heisenberg uncertainty rule in order to achieve superresolution. However, most of them uses fluorophore dyes, require a comprehensive image integration, or result in large devices with the need for qualified users and costly maintenance [1]-[3]. We investigate a simple idea for a different, novel type of microscope based on highly focused light source, which is an array of InGaN/GaN nanoLEDs [4]. Each single LED can be switched on and off independently, locally illuminating the sample, which is assumed to be in the optical near field. The shadow image of the object is then collected by the detectors (e.g. CMOS Single Photon Avalanche Photodiode SPAD). The microscope is designed to investigate a living tissue, thus on the top of the light engine a microfluidic chamber is integrated (modelled as SU8 and water domains). An advantage of this approach is the simplicity of sample preparation without fluorophore dyes and the possibility to develop lens-less, small, chip-based devices [4].

\section{A. System and methods}

In order to estimate and demonstrate the possible capabilities of the microscope, we performed full wave electromagnetic calculations by using Finite Difference Time Domain method (FDTD) implemented in the commercial software CST Studio.

The far field light intensity maps, resulting form pixel by pixel illumination of a specific object where calculated. In our analysis we focus on the normal outgoing (z) component

This work is being carried out in the European project ChipScope, funded by the European Union's Horizon 2020 research and innovation program under grant agreement no. 737089 . of the Poynting vector $P_{z}$, using the assumption of a large distance between the detector and the nanoLED array. As the structure (see Fig. 1) is anisotropic, we have investigated two perpendicular polarizations ( $\mathrm{x}$ and $\mathrm{y}$ ) of the dipole source and summed up the results.

The scheme of the modelled nanoLED array is shown in the Fig. 1. The radiation source was assumed as a single dipole located at the centre of the pixel in a distance $H_{2}=150 \mathrm{~nm}$ from the top surface of the LED. The operational wavelength was equal to $\lambda=450 \mathrm{~nm}$. We decided to analyse a very dense array of LEDs, i.e. with period $P=100 \mathrm{~nm}$ and LED width $w=50 \mathrm{~nm}$. In agreement with sampling theory the microscope should be able to resolve object of size equal to the twice the array period, thus in this case $200 \mathrm{~nm}$.

\section{B. Results and discussion}

Fig. 2 shows far field light intensity maps generated by the LED array, while illuminating various Au nanoparticle (NP) arrangements. The position and relative sizes of the objects is shown in the first row. In the second row of Fig. 2 we present the related normalised intensity patterns. One can notice a decrease in light intensity related to absorption in $\mathrm{Au}$ NPs, which clearly increases with the volume of the absorbing object. However the shapes of the NPs and the separation between two particle system is slightly blurred in the $x$ direction.

We expect that the patterns should reflect the symmetry of the objects and their position. Therefore, we analysed the symmetry of the patterns by drawing the map of differences (third row on the Fig. 2), i.e. the value of matrix elements is $x_{i, j}^{\prime}=\frac{\left(x_{i, j}-x_{L-i, L-j}\right)}{0.5\left(x_{i, j}+x_{L-i, L-j}\right)}$, where $L$ is the number of the last row/column. In the case of single NPs lying under the central pixel we confirm the symmetry of the signal across the $y=-x$ axis, but in the case of two NPs lying outside of the $x$ axis we obtain a visible deviation and therefore the signal resulting form two NPs can be distinguished from the signal coming from one single NP.

The complexity of the light intensity patterns can be explained by the complicated structure of the optical intensity distribution resulting from the activated pixels. The last two columns in the fourth row in Fig. 2 shows the electromagnetic field distributions in the image plane for the dense $(W=50$ 


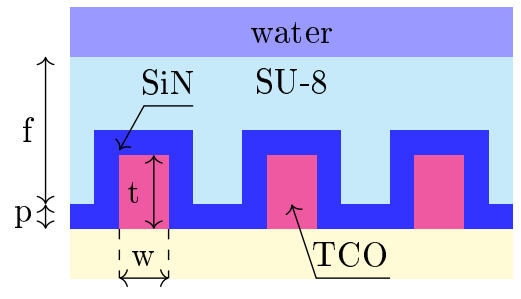

yz cross section

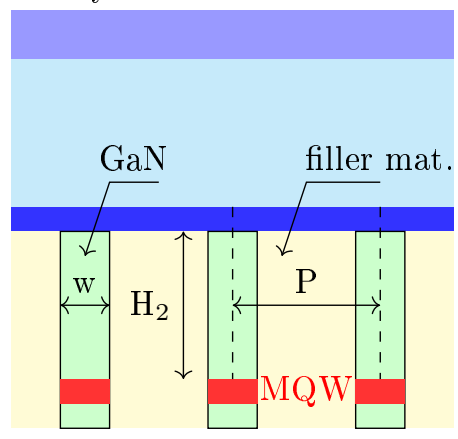

$\mathrm{xz}$ cross section rod architecture
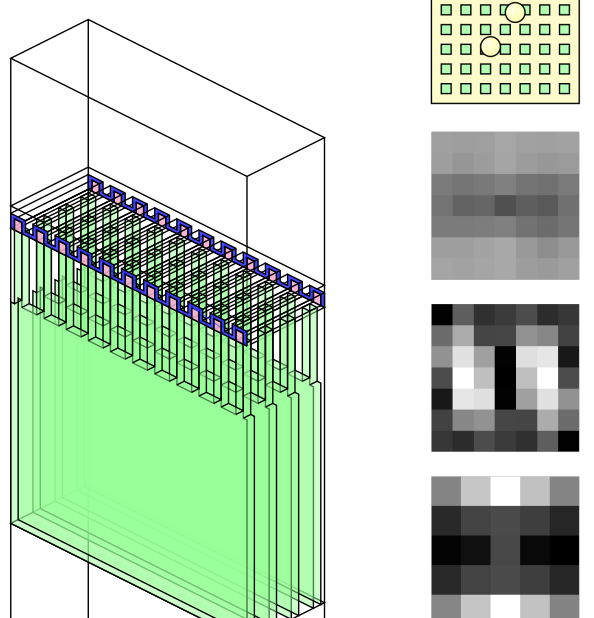

0.95111 .05

$\mathrm{P}_{\mathrm{z}}$ [a.u.]
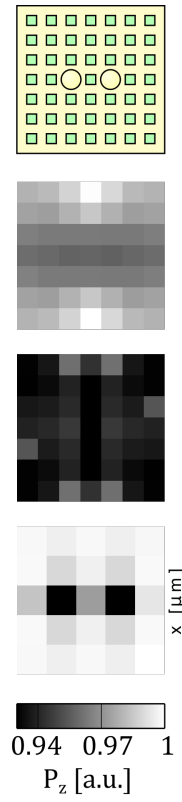

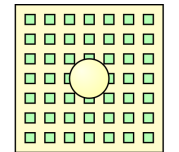

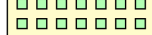
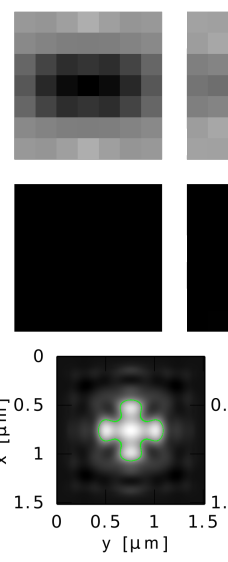

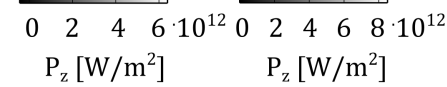

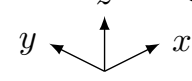

Fig. 1. Scheme of the nanoLED array architecture. In the simulations we have used array of size $13 \times 13$ pixels. The parameters used in the simulations were as follows: $w=50 \mathrm{~nm}, P=100 \mathrm{~nm}, H=4 \mu \mathrm{m}, H_{1}=1 \mu \mathrm{m}$, $H_{2}=150 \mathrm{~nm}, p=50 \mathrm{~nm}, t=150 \mathrm{~nm}, f=300 \mathrm{~nm}$. As a filler material, i.e. material located between the GaN domains we chose air, as we expect it to lead to the highest confinement of light inside GaN.

$\mathrm{nm}, P=100 \mathrm{~nm})$ and a sparse $(W=100 \mathrm{~nm}, P=300 \mathrm{~nm})$ LED array. For the dense array, due to the crosstalk between neighbouring pixels, the signal has multiple maxima, and its cross section strongly deviates from a circular shape. Full width at half maximum (FWHM), illustrated by green lines in the two lower right pnels in Fig. 2, is equal to few hundreds of nanometers. The crosstalk can be reduced by increasing the array period, by improving the guided mode confinement in the rod (e.g. by introducing anisotropic cladding on the rods [5]) or by increased optical isolation, e.g. by means of absorbing filling material. Indeed, the signal cross section for the sparse array shown in the rightmost bottom panel of Fig. 2 is much more restricted, and moreover shows higher intensity than the one for the dense array. On the left side in the fourth row of Fig. 2 we present the corresponding light intensity maps (left for the dense array and right for the sparse array) collected for two Au NPs laying along $x$ axis in one period distance. One can clearly see that for sparse array the sampling theory resolution limit is achievable.

\section{REFERENCES}

[1] L. Novotny and B. Hecht, "Principles of Nano-Optics," 2nd ed., Cambridge University Press, 2012.

[2] S. So, M. Kim, D. Lee, D. M. Nguyen, and J. Rho, "Overcoming diffraction limit: From microscopy to nanoscopy," Appl. Spectrosc. Rev. ,vol. 53, pp. 290-312 (2018).

[3] E. Sezgin, "Super-resolution optical microscopy for studying membrane structure and dynamics," J. Phys. Condens. Matter, vol. 29, pp. 273001, 2017.
Fig. 2. The first row, the schemes of the investigated Au nanoparticle arrangements. From the left: two NPs of radius $50 \mathrm{~nm}$ separated by the distance of one array pitch, i.e. $100 \mathrm{~nm}$ and rotated by $60^{\circ}$ in relation to $\mathrm{x}$ axis, two NPs of radius $50 \mathrm{~nm}$ separated by $100 \mathrm{~nm}$ laying along $\mathrm{x}$ axis, one NP of radius $50 \mathrm{~nm}$ located above central LED and one NP of radius $100 \mathrm{~nm}$ ones again placed above central pixel. The second row reports the far field light intensity collected by illuminating the above Au NPs arrangements with each single LED of the array. The third row, the related light pattern deviations from the symmetry across $y=-x$ axis. The fourth row, the light intensity patterns for dense $(W=50 \mathrm{~nm}, P=100 \mathrm{~nm}, 1 \mathrm{st})$ and sparse $(W=100 \mathrm{~nm}, P=300 \mathrm{~nm}, 2 \mathrm{nd})$ array in the case of two Au NPs laying along $\mathrm{x}$ axis in one period distance. The fourth row (3rd and 4th inset) signal xy cross section in the image plane for dense array (3rd) and sparse array (4th).

[4] J. Gülink, S. Bornemann, H. Spende, M. Auf der Maur, A. Di Carlo, J. D. Prades, H. S. Wasisto, and A. Waag, "InGaN/GaN nanoLED Arrays as a Novel Illumination Source for Biomedical Imaging and Sensing Applications," Proceedings, vol. 2, pp. 892, 2018.

[5] S. Jahani and J. Zubin, "Photonic skin-depth engineering," J. Opt. Soc. Am. B, vol. 32, pp. 1346-1353, 2015. 